Als Mittelwert ergab sich

$$
\begin{array}{ll}
36 \mathrm{GHz}: & \bar{\varepsilon}^{\prime}=2,54_{9} \pm 0,01_{2}, \\
42 \mathrm{GHz}: & \bar{\varepsilon}^{\prime}=2,55_{8} \pm 0,01_{5} .
\end{array}
$$

Die Genauigkeit in der Bestimmung der Dielektrizitätskonstanten hängt hauptsächlich von der Planparallelität der dielektrischen Platten ab. Die Abweichungen der $\varepsilon^{\prime}$-Werte, die aus drei Interferenzminima der gleichen Platte berechnet waren, lagen zwischen $\overline{\Delta \varepsilon^{\prime}}=0,006$ und 0,0026 .

Zum Vergleich wurde nach der von RoBerts und $\mathrm{H}_{\text {IPPEL }}{ }^{5}$ veröffentlichten Impedanzmethode bei $35 \mathrm{GHz}$ die Dielektrizitätskonstante zu $\overline{\varepsilon^{\prime}}=2,56 \pm 0,01$ gemessen.
Im Rahmen der angegebenen Fehlerbreiten dürfen beide Methoden im V-Band-Bereich als gleichwertig angesehen werden; die Übereinstimmung der Ergebnisse rechtfertigt die Fortsetzung der dielektrischen Untersuchungen bei Frequenzen über $36 \mathrm{GHz}$ mit der beschriebenen Reflektometer-Anordnung.

Der Deutschen Forschungsgemeinschaft sowie der Badischen Anilin- \& Soda-Fabrik A.G. Ludwigshafen danken wir für die Unterstützung dieser Arbeit. Der Firma Röhm \& Haas, Darmstadt, sind wir für die Überlassung und Bearbeitung der Plexiglasplatten zu Dank verpflichtet.

5 S. Roberts u. A. v. Hippel, J. Appl. Phys. 17, 610 [1946].

\section{ESR-Untersuchungen zur Eigenradiolyse tritiummarkierter Amino- und Dicarbonsäuren}

Von G. Lassmann und W. Damerau

Institut für Biophysik der Deutschen Akademie der Wissenschaften zu Berlin*

und H. Herzmann

Institut für Angewandte Isotopenforschung der Deutschen Akademie der Wissenschaften zu Berlin *

(Z. Naturforschg. 19 a, 661-662 [1964] ; eingegangen am 10. April 1964)

Bei Elektronenspinresonanz-(ESR)-Untersuchungen über die Radiolyse organischer Moleküle im Festkörpergitter erfolgte die Bestrahlung mit energiereicher Strahlung bisher in der Regel von außen. Die Befunde vieler Arbeiten der letzten Jahre haben ergeben, da $\beta$ in fast allen organischen Kristallen nach hinreichend intensiver äußerer Bestrahlung mit $\gamma$ - oder Röntgen-Strahlen paramagnetische Zentren (meistens Radikale) gebildet werden, die eine Zeitlang beständig sind. Es war deshalb anzunehmen, daß auch in Verbindungen, die mit radioaktiven Isotopen markiert sind, durch die Zerfallsenergie Radikale erzeugt werden.

Die vorliegenden Untersuchungen wurden begonnen, um das Studium strahleninduzierter Radikale mit der ESR auch auf die Eigenradiolyse markierter Verbindungen auszudehnen. Durch die Wahl der Aminosäuren als Versuchsobjekt wurde der Frage nach der Strahlenschädigung durch Inkorporation radioaktiver Nuklide Rechnung getragen. Darüber hinaus erschließt die Kenntnis der Stufen, über die sich eine markierte Substanz zersetzt, Möglichkeiten zur Verringerung der Eigenradiolyse durch geeignete Aufbewahrungsbedingungen.

Von Квон et al. wurde bei ESR-Messungen Tritiumwasser als $\beta$-Strahler der Substanz beigemischt, um

* Berlin-Buch, Lindenberger Weg 70.

1 J. Кroh, B. C. Green u. J. W. T. Spinks, Nature, Lond. 189 , 655 [1961].

2 W. Damerau, G. Lassmann u. H.-G. Thom, Z. Phys. Chem. (Leipzig) 223, 99 [1963]. die unerwünschten Glasresonanzen bei äußerer Bestrahlung zu vermeiden ${ }^{1}$.

Eine Eigenbestrahlung durch direkte ${ }^{3} \mathrm{H}$-Substitution der zu untersuchenden Substanz wurde kürzlich vorgeschlagen, und es konnten ESR-Signale von tritierten Substanzen nachgewiesen werden ${ }^{2}$. Im weiteren soll die Ursache der Radikalbildung in direkt mit ${ }^{3} \mathrm{H}$-markierten Molekülen näher betrachtet werden. Dazu wurden an ${ }^{3} \mathrm{H}$-substituierten Verbindungen quantitative ESRMessungen begonnen.

Zur Untersuchung gelangten feste polykristalline Amino- und Dicarbonsäuren sowie Asparagin (siehe Tabelle), die mit der Gasaustauschmethode nach WILzВACH ${ }^{3}$ bzw. mit Tritiumwasser tritiert wurden.

Die tritiumhaltigen Substanzen wurden mehrere Monate bei Zimmertemperatur aufbewahrt, bis sich ${ }^{3} \mathrm{H}-\beta$-Zerfälle in genügend großer Anzahl ereignet hatten. Darauf wurden die Proben in einem X-Band-ESRSpektrometer gemessen und die Spektren quantitativ ausgewertet.

Zur Radikalausbeutemessung wurde unmittelbar nach jeder Messung das Spektrum einer ZuckerkohleEichprobe bekannten Spingehalts unter gleichen Spektrometerbedingungen im $H_{102}$-Rechteckresonator aufgenommen. Nach Integration der 1. Ableitung der Spektren von Meß- und Eichprobe mit einem mechanischen Integrator wurde aus dem Verhältnis der Flächengewichte die Absolutzahl der Radikale in der Meßprobe ermittelt. Die Eichung der Zuckerkohle erfolgte gegen eingewogene Mikromengen von festem DPPH (Titer 95\%). Um eine unterschiedliche Veränderung der Resonatorgüte durch die beiden Proben zu erfassen, haben wir sowohl bei Zuckerkohle als auch bei DPPH dieselbe Menge einer $\mathrm{ZnS} / 0,05 \% \mathrm{Mn}^{2+}$-Probe mitgemessen ${ }^{4}$. Diese Substanz zeigt 6 starke Hyperfeinstrukturlinien vom $\mathrm{Mn}^{2+}$-Ion, wobei die Mitte bei $g=2,00$ in einer Breite von ca. $30 \mathrm{Gauß}$ frei bleibt. Die Amplituden der

3 K. E. Wilzbach, J. Amer. Chem. Soc. 79, 1013 [1957].

${ }^{4}$ Für die freundliche Überlassung dieser Probe danken wir Herrn Dr. Ulbert aus Prag. 


\begin{tabular}{|c|c|c|c|c|c|c|}
\hline Nr. & Substanz & ${ }^{3} \mathrm{H}$-Markierung & $\begin{array}{l}\text { Spezifische } \\
\text { Aktivität } \\
(\mathrm{mC} / \mathrm{g})\end{array}$ & $\begin{array}{c}{ }^{3} \mathrm{H}-\text { Zerfälle zwischen } \\
\text { Markierung und } \\
\text { ESR-Messung }\end{array}$ & $\begin{array}{l}\text { Radikalanzahl } \\
\text { je Probe }\end{array}$ & $\begin{array}{l}\text { Anzahl stabili- } \\
\text { sierter Radikale } \\
\text { pro }{ }^{3} \mathrm{H} \text {-Zerfall }\end{array}$ \\
\hline $\begin{array}{r}1 \\
2 \\
3 \\
4 \\
5 \\
6 \\
7 \\
8 \\
9 \\
10\end{array}$ & $\begin{array}{l}\text { Glycin } \\
\text { DL- } \alpha \text {-Alanin } \\
\text { L-Cystein } \cdot \text { HCl } \\
\text { L-Cystein } \\
\text { DL-Tryptophan } \\
\text { L-Leucin } \\
\text { Asparagin } \\
\text { Bernsteinsäure } \\
\text { Bernsteinsäure } \\
\text { Adipinsäure }\end{array}$ & 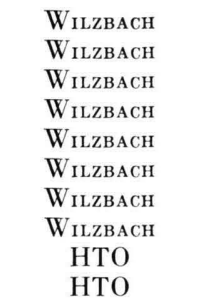 & $\begin{array}{c}0,23 \\
328 \\
3,24 \\
223 \\
13,6 \\
592 \\
16,6 \\
243 \\
121 \\
126\end{array}$ & $\begin{array}{l}1,5 \cdot 10^{13} \\
7,9 \cdot 10^{15} \\
1,9 \cdot 10^{14} \\
6,8 \cdot 10^{15} \\
4,6 \cdot 10^{14} \\
9,2 \cdot 10^{15} \\
1,2 \cdot 10^{15} \\
1,4 \cdot 10^{16} \\
9,1 \cdot 10^{14} \\
6,4 \cdot 10^{14}\end{array}$ & $\begin{array}{l}<10^{15} \\
7,4 \cdot 10^{17} \\
1,7 \cdot 10^{16} \\
1,1 \cdot 10^{17} \\
1,3 \cdot 10^{15} \\
1,1 \cdot 10^{17} \\
5,2 \cdot 10^{16} \\
2,5 \cdot 10^{16} \\
3,0 \cdot 10^{16} \\
1,9 \cdot 10^{16}\end{array}$ & $\begin{array}{c}\text { kein ESR-Signal } \\
94 \\
90 \\
16 \\
2,8 \\
12 \\
43 \\
1,8 \\
33 \\
30\end{array}$ \\
\hline
\end{tabular}

Tab. 1. Radikalausbeuten in mit Tritium direkt markierten Verbindungen.

beiden inneren Linien dienten als Vergleichsmaßstab für eine Änderung der Bedämpfung des Resonators durch die Probe.

Die ESR-Messungen ergaben bis auf ${ }^{3} \mathrm{H}$-Glycin von allen untersuchten ${ }^{3} \mathrm{H}$-markierten Verbindungen gut meßbare Radikalkonzentrationen von $1 \cdot 10^{15}-7 \cdot 10^{17}$ Radikalen je Probe. Eine Gegenüberstellung der Radikalanzahl in der Probe und der Anzahl ${ }^{3} \mathrm{H}$ - $\beta$-Zerfälle während der Zeit zwischen Markierung und ESR-Messung ist in der Tabelle gezeigt. Der Vergleich ergibt, daß die Anzahl der gebildeten und stabilisierten Radikale erheblich größer ist als die Zahl der $\beta$-Zerfälle. Diese Befunde lassen schließen, daß es sich bei den gefundenen paramagnetischen Zentren nicht um solche Radikale handeln kann, die durch Elementumwandlung ${ }^{3} \mathrm{H} \rightarrow{ }^{3} \mathrm{He}$ beim Zerfall an der Markierungsstelle entstehen könnten. Ihre Anzahl dürfte nicht größer als die Zahl der ${ }^{3} \mathrm{H}$ - $\beta$-Zerfälle sein. Die Hauptursache der Radikalbildung kann nur in einer Bestrahlung der Substanz von „innen her“ durch Einwirkung der $\beta$-Teilchen gesehen werden. Dafür spricht auch die in vielen Fällen beobachtete Ähnlichkeit der Spektren tritierter Verbindungen mit den nach $\gamma$ - oder Röntgen-Bestrahlung erhaltenen Spektren derselben Substanz ${ }^{5}$.

Bei einer Strahlenschädigung organischer Moleküle durch Eigenbestrahlung infolge Zerfalls radioaktiver Isotope unterscheiden B BYLY und $\mathrm{W}_{\text {EIGEL }}{ }^{6}$ hauptsächlich zwei Typen der primären Strahlenwirkung, die zum

5 G. Lassmann, H. Herzmann u. W. Damerau, unveröffentlicht.

6 R. J. Bayly u. H. Weiged, Nature, Lond. 188, 384 [1960].
Zerfall oder zur Umwandlung der Moleküle führen. Der „innere Effekt“ wird verursacht durch die Elementumwandlung beim radioaktiven Zerfall der Markierungsatome, während der „äußere Effekt“ durch Wechselwirkung der Moleküle mit den emittierten Kernpartikeln hervorgerufen wird.

Für die Ursache der Radikalbildung in tritiummarkierten Molekülen konnte sowohl die Umwandlung eines am Molekül gebundenen ${ }^{3} \mathrm{H}$-Atoms in ein nichtbindendes Edelgasatom als auch die Bestrahlung durch die beim Zerfall emittierten $\beta$-Teilchen angenommen werden. LAMBE $^{7}$ folgerte aus ESR-Untersuchungen an festem Tritium bei $4,2{ }^{\circ} \mathrm{K}$, daß einerseits durch spezifische ${ }^{3} \mathrm{H}$-Markierung eine bestimmte Erzeugung von Defekten möglich sei, andererseits aber die übrige Substanz durch die $\beta$-Teilchen nicht direkt beschädigt würde.

Ein Vergleich mit unseren Befunden zeigt, daß eine Verallgemeinerung über das Modell des festen Tritiums hinaus auf ${ }^{3} \mathrm{H}$-markierte Verbindungen nicht ohne weiteres erlaubt ist. Die $\beta$-Partikel spielen bei ${ }^{3} \mathrm{H}$-markierten Amino- und Dicarbonsäuren die dominierende Rolle bei der Radikalbildung durch die Eigenradiolyse.

Eine ausführliche Publikation erfolgt später.

Herrn Prof. Dr. A. Lösche und Herrn Dr. H. Pupke möchten wir für das Interesse am Fortgang der Arbeit und den Herren Ing. J. Engel, Ing. N. Klimes, M. Dobberphul und Fräulein G. KLEıN für ihre Mitarbeit danken.

7 J. Lambe, Phys. Rev. 120, 1208 [1960]. 